\title{
Paper
}

Int'I J. of Aeronautical \& Space Sci. 18(4), 719-728 (2017)

DOI: http://dx.doi.org/10.5139/JASS.2017.18.4.719

\section{Impact Angle Control Guidance Synthesis for Evasive Maneuver against Intercept Missile}

\section{Y. H. Yogaswara*}

Korea Advanced Institute of Science and Technology, Daejeon 34141, Republic of Korea

Department of Research and Development of Indonesian Air Force, Bandung 40174, Indonesia

\section{Seong-Min Hong** and Min-Jea Tahk***}

Korea Advanced Institute of Science and Technology, Daejeon 34141, Republic of Korea

\author{
Hyo-Sang Shin**** \\ Cranfield University, Bedford MK43 0AL, United Kingdom
}

\begin{abstract}
This paper proposes a synthesis of new guidance law to generate an evasive maneuver against enemy's missile interception while considering its impact angle, acceleration, and field-of-view constraints. The first component of the synthesis is a new function of repulsive Artificial Potential Field to generate the evasive maneuver as a real-time dynamic obstacle avoidance. The terminal impact angle and terminal acceleration constraints compliance are based on Time-to-Go Polynomial Guidance as the second component. The last component is the Logarithmic Barrier Function to satisfy the field-of-view limitation constraint by compensating the excessive total acceleration command. These three components are synthesized into a new guidance law, which involves three design parameter gains. Parameter study and numerical simulations are delivered to demonstrate the performance of the proposed repulsive function and guidance law. Finally, the guidance law simulations effectively achieve the zero terminal miss distance, while satisfying an evasive maneuver against intercept missile, considering impact angle, acceleration, and field-of-view limitation constraints simultaneously.
\end{abstract}

Key words: Missile guidance, Evasive maneuver, Impact angle control, Artificial potential field

\section{Introduction}

Since an Integrated Air Defense Systems (IADS) [1] have been sophistically developed, a countermeasure action to counteract the IADS becomes a significant consideration in a missile design. Surface attack missiles, which are launched from air or surface platforms to attack designated surface targets also need advanced solutions to respond the threats of IADS. Guidance system design for surface attack missile in this high threat environment is challenging since the attacking missile must be delivered to its target, while maximizing the survivability from the IADS. A proper guidance laws to generate an evasive maneuver against intercept missiles are rarely published in open literature. On the contrary, the evasive maneuver of manned aircraft against intercept missile has been studied extensively. Those evasive strategies are based on continuously changing maneuver direction such as barrel roll and the Vertical-S maneuver [2, p. 120] or weaving maneuver [2, Ch. 27]. The strategies are not adequate to be implemented into homing missiles or unmanned aerial vehicles (UAVs) due to several reasons, i.e.: the movement can be easily predicted, the maneuver might exceed seeker's fieldof-view (FOV) limitation, and the missile fails to satisfy zero terminal miss distance.
This is an Open Access article distributed under the terms of the Creative Commons Attribution Non-Commercial License (http://creativecommons.org/licenses/by$\mathrm{nc} / 3.0 /$ ) which permits unrestricted non-commercial use, distribution, and reproduction in any medium, provided the original work is properly cited. (c) * Ph. D Student, Research Officer

** Ph. D Student

*** Professor, Corresponding author: mjtahk@kaist.ac.kr

$* * * *$ Associate Professor 
An optimal evasive maneuver for subsonic Anti-Ship Missiles (ASM) has been studied against Close-in Weapon System (CIWS) [3]. The CIWS is a common short-range IADS for naval ships that are usually equipped with cannon as the defensive weapon. Since the ASM moves with constant acceleration at any moment, the CIWS is able to aim at the predicted intercept position. In [3], the authors have defined a time-varying weighted sum of the inverse of the aiming errors as a cost function which to be minimized. Inspired by the study above, a direct optimization technique using Co-Evolutionary Augmented Lagrangian Method (CEALM) was applied in [4], which the capturability analysis is proven using Lyapunov-like approach. Both studies in [3] and [4] generate a type of barrel roll evasive maneuver, however, a large barrel roll maneuver could increase the miss distance at the terminal time.

As studied in [5], the typical CIWS's cannon system has limitations due to single-threat-engagement capability and fire at a predicted intercept point of the threat's trajectory. This limitation motivates a new guidance law by using salvo attack where multiple missiles are launched and guided to arrive at a stationary target at the same desired time. The guidance law adopting this idea has been firstly proposed by Jeon et al. in [6] and called as Impact Time Control Guidance (ITCG). To control the impact time, the ITCG suggests an additional loop in a closed form solution based on the linear formulation of traditional proportional navigation guidance (PNG) law. The ITCG has been also proposed in [7] by introducing a virtual leader approach. Sliding mode control (SMC) method for ITCG has been adopted in [8]. A Lyapunov-based law is the latest method proposed in [9] for ITCG and simultaneous arrival.

Even though the ITCG has been broadly studied, the concept of salvo attack for simultaneous impact time by exploiting the limitation of CIWS is less effective. In fact, if we may be allowed for a little exaggeration, it is not applicable anymore. This knowledge is exposed since CIWS or IADS introduces intercept missiles to enhance the previous cannon system. The intercept missile has advanced capability of multi-target engagement, longer intercept range, and proximity warhead detonation for a higher probability of kill. Regarding those new developments, this paper proposes a new guidance law, which maximizes survivability of an attack missile with respect to the threat of advanced CIWS or IADS. In order to neutralize the threat of intercept missile of enemy's IADS, our attack missile must have an evasive maneuver capability to counteract the interception. In addition, the attack missile must satisfy an impact angle constraint and zero terminal acceleration for maximizing warhead detonation effect on the target. FOV limitation of the attack missile is also considered to ensure the seeker locks on the target.

Due to its elegant concept and simple computation, the evasive maneuver of the attack missile in this paper is based on the concept of Artificial Potential Field (APF). The APF was introduced by Krogh [10] and Khatib [11] for mobile robots by defining functions of goal attractive potentials and obstacle repulsive potentials. Inherent problems of APF were systematically identified by Koren and Borenstein in [12] due to a trap situations caused by local minima, no passage between close space obstacle, and oscillation. In addition, Goal Non-reachable with Obstacle Nearby (GNRON) problem was also recognized and solved by Ge and Cui in [13]. Modifications of APF for moving obstacles have been also proposed in [14]-[16]. Nevertheless, those modifications cannot be implemented to the missile problem since the modified potential functions require a deceleration variable to reduce velocity when facing the obstacle. Eventually, inspired by Chen et al. in [17], this paper proposes a new repulsive function that effective for the missile problem and overcomes the GNRON problem at the same time. The primary limitation of APF on trap situation due to local minima will not be an issue in this evasive maneuver scenario since the problem is one-on-one engagement with relatively free dead-end trajectory, e.g. U-shaped obstacle.

Regarding the concept of APF, instead of the conventional attractive potential function, this paper implements an impact angle control guidance (IACG) method as the attractive goal. The IACG attacks the weak spot of a target to increase warhead detonation effect and maximize its probability of kill towards the target. A generalized formulation of energy minimization had been proposed in [18] to achieve IACG. A PNG-based was proposed in [19] for capturing all possible terminal impact angle. Lyapunovbased pursuit guidance was introduced in [20] to reduce the angle between the velocity and the distance vector. A combination of differential game and sliding mode control were also proposed in [21]. The Time-to-go Polynomial Guidance (TPG) as proposed by Lee et al. in [22] which treat the guidance command as a function of time-to-go is the most suitable to be adopted in this paper. In addition to the impact angle constraint, the TPG also satisfies the zero terminal acceleration to minimize the terminal angle-ofattack (AOA) for effective impact angle, and zero terminal lateral velocity to minimize zero effort miss.

In order to improve the practicality on the physical concern of proposed guidance law, the limitation on actuator's acceleration command is considered in this paper. Moreover, the seeker look angle is also limited to be inside the FOV in order to ensure the missile seeker not losing its target. 
Switching logic was implemented in [23] by switching the guidance law from ITCG of [6] to the second law of constant look angle guidance command, when the seeker look angle exceeds the FOV limit. A rule of the cosine of a weighted leading angle in the biased term was used in [24] to ensure the ITCG was satisfied without violating the FOV constraint during an engagement. Switching logic to constant seeker look angle was also investigated in [25] for optimal IACG of a missile with a strap-down seeker. Without applying the switching logic, SMC was used in [26] to satisfy IACG by implementing a control Integral Barrier Lyapunov Function (iBLF) to design the reaching law. The implementation of iBLF inspires this paper to apply a simpler internal penalty function known as Logarithmic Barrier Function (LBF) to limit the look angle.

This paper is organized as follows. Section 2 presents the new proposed repulsive potential functions of APF with its properties. In Section 3, the equations of motion, APF, TPG, LBF and all corresponding problem formulation are synthesized to generate a single acceleration command of proposed guidance law. Guidance characteristics, analysis, and solutions are presented in Section 4 by numerical simulation at a particular missile engagement scenario. The simulations successfully demonstrate the effectiveness of the potential function and guidance law. Finally, concluding remarks and future works are given in Section 5.

\section{Proposed Repulsive Potential Function}

The repulsive potential function that is implemented on mobile robots, robot manipulators, and UAVs are broadly based on the Khatib [11] or Ge and Cui [13]. However, those repulsive potential functions are not suitable to be used in missile engagement problem due to its potential characteristics. The main properties of those functions is a steep slope of repulsive potential, which the potential values ascent sharply approaching the obstacle position. This property is acceptable for mobile robots since it has the capability of deceleration when approaching the obstacle, stop the movement, and reroute its path. Conversely, the missiles engagement scenario does not recognize those capabilities.

The repulsive potential function for missile engagement scenario needs a gradual ascent of potential approaching the obstacle to anticipate the intercept in advance. The gradual and gentle ascent of repulsive potential has been introduced for UAV path planning by Chen et al. in [17]. Unfortunately, this function does not consider the GNRON problem, which drives a failure to achieve the goal position. The failure is generated by shifting out the global minimum from the goal position when the goal is within the influence distance of the obstacle.

A new repulsive potential function of APF is constructed and proposed to generate a gradual ascent of potential approaching the obstacle and solve the GNRON problem at the same time. Assuming a generic problem of a point masses vehicle, goal, and obstacle, which the vehicle moves in a two-dimensional (2-D) space. The vehicle position in the workspace is denoted by $\mathbf{q}=\left[\begin{array}{ll}x_{A} & y_{A}\end{array}\right]^{T}$. The repulsive potential $U_{\text {rep }}(\mathbf{q})$ at each vehicle position is defined by considering the relative distance of goal position into Chen's equation as follow

$$
\begin{aligned}
& U_{\text {rep }}(\mathbf{q})=\left\{\begin{array}{cc}
\varepsilon e^{-\zeta d_{o b s}} d_{\text {goal }}, & \text { if } d_{o b s t} \leq d_{o} \\
0, & \text { if } d_{o b s t}>d_{o},
\end{array}\right. \\
& d_{\text {goal }}=\left\|\mathbf{q}_{\text {goal }}-\mathbf{q}\right\| \\
& d_{\text {obst }}=\left\|\mathbf{q}_{\text {obst }}-\mathbf{q}\right\|,
\end{aligned}
$$

where $d_{\text {obst }}, d_{\text {goal }}, d_{0}, \varepsilon$, and $\zeta$ are the minimal distance between the vehicle and the obstacle, the distance between the vehicle and the goal, the distance of influence of the obstacle, and both are positive design parameter gains, respectively. This proposed function ensures the repulsive potential approaches zero as the vehicle approaches the goal and finally the goal position will be the global minimum of total potential.

The effectiveness of the proposed repulsive potential function is demonstrated in a case on one-dimensional (1-D) space as shown in Fig. 1. The vehicle $\mathbf{q}=\left[\begin{array}{ll}x_{A} & 0\end{array}\right]^{T}$ is moving along x-axis toward the goal $\mathbf{q}_{\text {goal }}=\left[\begin{array}{ll}4 & 0\end{array}\right]^{T}$ while avoiding the obstacle $\mathbf{q}_{\text {obst }}=\left[\begin{array}{ll}0 & 0\end{array}\right]^{T}$. Assuming the distance of influence of the obstacle $d_{0}=6$, the GNRON problem of the predecessor function as mentioned by Chen et al. in [17] is demonstrated in the first plot series. Since the goal position near the obstacle, the generated repulsive potential is large enough to create the non-reachable goal. This problem takes place since the goal position is affected by the obstacle and

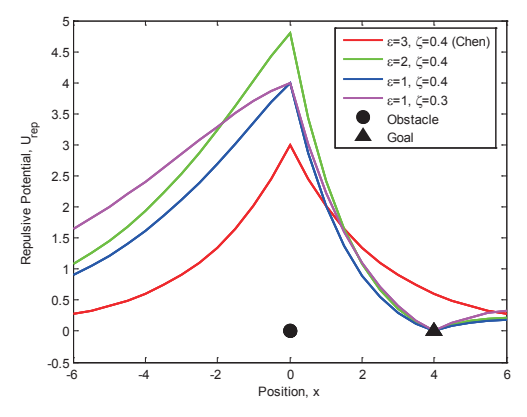

Fig. 1. Repulsive potential function in a 1-D space 
drive non-zero potential at the goal. Moreover, the potentials are evenly distributed to the right and the left side of the obstacle neglecting the goal. In the same case assumption, the new proposed function shows significant improvement to handle the GNRON problem. The plot of three different combinations of scaling gains maintains the minimum of the potential at the goal position and the maximum of the potential at the obstacle position. Furthermore, the scaling gains show the freedom to control the properties of repulsive potential. The higher value of $\varepsilon$, the higher peak value of the potential. The higher value of $\zeta$, the steeper ascent of potential approaching the obstacle.

The corresponding repulsive force is given by the negative gradient of the repulsive potential. According to Eq.(1), when the vehicle is not at the goal, i.e., $\mathbf{q} \neq \mathbf{q}_{\text {goal }}$, the repulsive force is given by

$$
\begin{aligned}
& F_{\text {rep }}(\mathbf{q})=-\nabla\left(U_{\text {rep }}(\mathbf{q})\right) \\
& F_{\text {rep }}(\mathbf{q})= \begin{cases}F_{\text {repObst }} \mathbf{n}_{\text {obst }}+F_{\text {repGoal }} \mathbf{n}_{\text {goal }}, & \text { if } d_{\text {obst }} \leq d_{o} \\
0, & \text { if } d_{\text {obst }}>d_{o},\end{cases} \\
& F_{\text {repobst }}=\varepsilon \zeta d_{\text {goal }} e^{-\zeta d_{\text {obst }},}, \\
& F_{\text {repGoal }}=\varepsilon e^{-\zeta d_{\text {obst }},}
\end{aligned}
$$

where $\mathbf{n}_{\text {obst }}=\nabla d_{\text {obst }}$ and $\mathbf{n}_{\text {goal }}=-\nabla d_{\text {goal }}$ are unit vectors pointing from the obstacle to the vehicle and from the vehicle to the goal, respectively. Those unit vectors play an

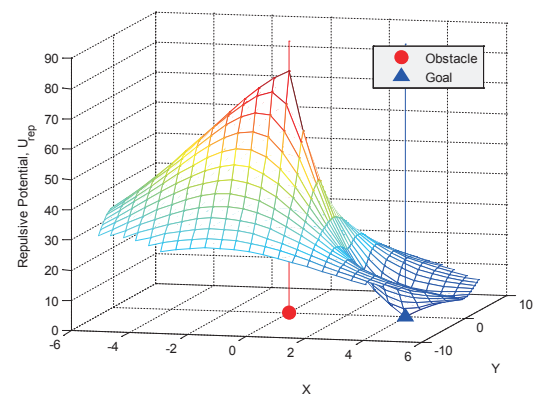

important role since the $\mathbf{n}_{\text {obst }}$ repulses the vehicle away from the obstacle and the $\mathbf{n}_{\text {goal }}$ attracts the vehicle towards the goal.

To elaborate the properties of the force field, the case on Fig. 1 is developed into 2-D space, which the scaling gains are defined as $\varepsilon=20$ and $\zeta=0.3$. The repulsive potential field and repulsive force field of the vehicle at every position in a 2-D space are depicted in Fig. 2. The repulsive potential field keeps the goal as the global minima and the potential peak at the obstacle. The repulsive potential force represents the potential as a positive divergent vector field outward the obstacle and a negative divergent vector field inward the goal. Intuitively, the vehicle that affected by this vector field will be repulsed by the obstacle and attracted to the goal.

Regarding the implementation of the new repulsive function into missile evasive maneuver, some nomenclatures are adjusted. The vehicle of interest, the obstacle and the goal are defined as the attack missile, the intercept missile, and the target, respectively.

\section{Guidance Synthesis}

Consider a two-dimensional homing guidance scenario as shown in the left illustration of Fig. 3. The friendly attack missile has a constant velocity $V_{A}$ heading to enemy's stationary target while avoiding enemy's intercept missile,

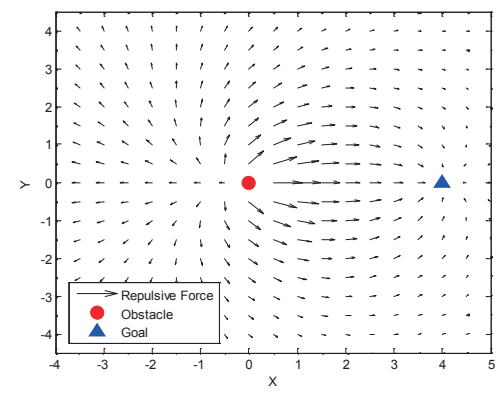

Fig. 2. Repulsive potential field (left) and repulsive force field (right) in a 2-D space
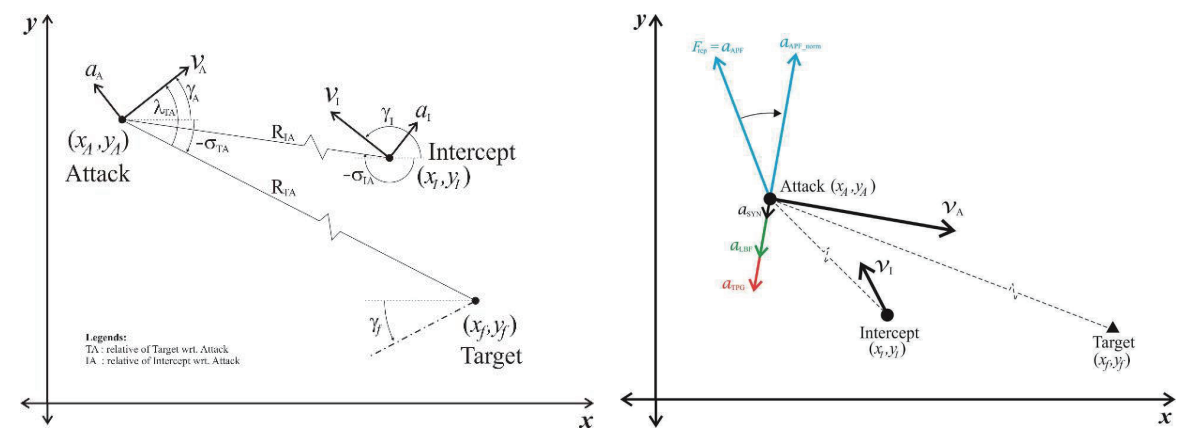

Fig. 3. Guidance geometry (left) and guidance synthesis of acceleration command vector (right) 
which has a constant velocity $V_{I}$. Acceleration command $a$ is perpendicular to velocity vector to change the flight path angle $\gamma$ of each missile. The position of the attack missile, the intercept missile, and the target are denoted as $\left(x_{A}, y_{A}\right),\left(x_{I}, y_{I}\right)$, and $\left(x_{T}, y_{T}\right)$, respectively. Their relationships are denoted as

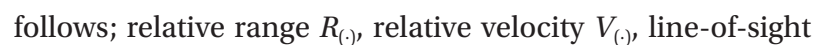

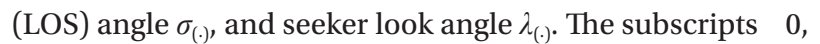
$f, A, I, T A, I A$ denote the initial time, terminal time, attack, intercept, relationship of the attack missile regarding the target, and the attack missile regarding the interceptor, respectively.

The equation of motion in this homing problem for both attack and intercept missile in inertial frame are generally given by

$$
\begin{aligned}
& \frac{d x}{d t}=V \cos \gamma \\
& \frac{d y}{d t}=V \sin \gamma \\
& \frac{d \gamma}{d t}=\frac{a}{V} .
\end{aligned}
$$

With its boundary conditions are defined as follows:

$$
\begin{array}{lll}
x\left(t_{0}\right)=x_{0} & y\left(t_{0}\right)=y_{0} & \gamma\left(t_{0}\right)=\gamma_{0} \\
x\left(t_{f}\right)=x_{f} & y\left(t_{f}\right)=y_{f} & \gamma\left(t_{f}\right)=\gamma_{f} .
\end{array}
$$

The guidance law is derived by using small angle approximation of AOA, that the velocity vector and body orientation nearly have the same value. Hence, the seeker look angle of attack missile toward the target can be approximated as subtraction of flight path and LOS angle

$$
\lambda=\gamma-\sigma .
$$

Recalling the right illustration of Fig. 3, the total acceleration command of the guidance law $a_{A}=a_{S Y N}$, which a synthesis of three components is simply

$$
a_{S Y N}=a_{T P G}+a_{A F P}+a_{L B F} .
$$

This formulation is described as follow. Principally, the guidance synthesis implements the APF concept by defining the attractive potential to be achieved and repulsive potential to be avoided. Rather than implementing classical attractive force function of APF, the acceleration command of TPG $a_{T P G}$ is preferred to achieve zero terminal miss distance at designated terminal impact angle and zero terminal acceleration. The new proposed repulsive force function of APF performs as the second component to generate the evasive maneuver. Recalling Eq. (3), the acceleration command of APF is taken from the proposed repulsive potential force $a_{A P F}=F_{r e p}(\mathbf{q})$. Once the intercept missile approaching, the repulsive force is generated as $a_{A P F}$ and producing a new resultant vector of $\left(a_{T P G}+a_{A F P}\right)$ avoiding the interceptor. Finally, acceleration command of LBF $a_{L B F}$ is also generated when the seeker look angle close to its FOV limit by compensating the exceeding acceleration command. Through this model, the synthesized guidance law propose a responsive approach to achieve an effective evasive maneuver while satisfying zero miss distance, terminal impact angle, zero terminal acceleration, and FOV limitation.

\subsection{Time-to-Go Polynomial Guidance}

The TPG demonstrates an effective IACG not only its ability to satisfies the terminal impact angle, but also satisfies the zero terminal acceleration to minimize the terminal AOA for precise impact angle, and zero terminal lateral velocity to minimize zero effort miss. Recalling the TPG on [22], the missile acceleration command $a_{T P G}$ and the estimation of time-to-go $t_{g o}$ for the curved path of the attack missile can be formulated as follows:

$$
\begin{aligned}
a_{T P G}(t)= & -\frac{V_{A}}{t_{g o}}\left[-(m+2)(n+2) \sigma(t)+(m+n+3) \gamma_{A}(t)\right. \\
& \left.+(m+1)(n+1) \gamma_{f}\right], \\
t_{g o}= & \frac{R}{V_{A}}\left\{1+P_{1}\left[\left(\frac{1}{2}\left(\gamma_{A}-\gamma_{f}\right)-P_{2}\left(\sigma_{T A}-\gamma_{f}\right)\right)^{2}\right.\right. \\
& \left.\left.+P_{3}\left(\gamma_{A}-\gamma_{f}\right)^{2}\right]-\frac{1}{2}\left(\sigma_{T A}-\gamma_{f}\right)^{2}\right\}, \\
P_{1}= & \frac{1}{(2 m+3)(2 n+3)(m+n+3)} \\
P_{2}= & (m+2)(n+2) \\
P_{3}= & \left(m+\frac{3}{2}\right)\left(n+\frac{3}{2}\right)
\end{aligned}
$$

where $m$ and $n$ denote the guidance gains which are chosen to be any positive real values following $n>m>0$ for zero terminal acceleration. If $n=1$ and $m=0$, the performance of applied TPG results are identical to the optimal guidance laws with terminal impact constraints, but without zero terminal acceleration as studied in [18]. Higher values of $\mathrm{m}$ and $\mathrm{n}$ gains will not only satisfy desired impact angle but also produce the zero terminal acceleration to avoid saturating commands and sufficiently small terminal AOA to increase the lethality of the warhead.

On the other hand, the enemy's intercept missile applies a Pure Proportional Navigation (PPN) in order to intercept the attack missile. The PPN is chosen due to its natural characteristics in a practical sense as concluded by Shukla and Mahapatra in [27]. Referring to the literature, the acceleration command for the intercept missile is formulated as 


$$
\begin{gathered}
a_{I}=-N V_{I} \dot{\sigma}_{I A} \\
\dot{\sigma}_{I A}=\frac{R_{A I} \times V_{A I}}{R_{A I} \cdot R_{A I}},
\end{gathered}
$$

where the navigation constant is defined as $N=3$.

\subsection{Logarithmic Barrier Function}

In a real application, the target should be located inside the FOV of the attack missile, and it is important to keep the seeker look angle from exceeding the limitation. Regarding the look angle on conventional PNG that decreases to zero as the missile approaches its target, the proposed guidance law is intended to achieve an additional capability. This capability generates an uncommon trajectory that increases the look angle up to exceeds the FOV. When the missile fails to lock on the target, it leads the missile into a huge miss distance and unsatisfied constraints at the terminal phase. Introducing the FOV limit as the barrier $\mathrm{b}$ and the barrier parameter $\mu$, the final component in Eq. (8) can be easily derived by using LBF. Implementing the characteristics of LBF into a compensated acceleration command ensures the command increases to actuator's limitation $a_{\lim }$ as the current look angle approaching the barrier and keep the seeker look angle inside the FOV in a simple way. Acceleration command in component of LBF can be formulated as

$$
a_{L B F}=\left\{\begin{array}{ll}
a_{\lim } & \text { if } \lambda \geq b \\
\mu \log (b-|\lambda|) & \text { if } b>\lambda \geq\left(b-10^{\circ}\right) \\
0 & \text { if } \lambda<\left(b-10^{\circ}\right)
\end{array} .\right.
$$

Finally, by substituting Eqs. (3), (9), and (13) into (8), the total acceleration command of attack missile is synthesized as a new proposed guidance law.

\subsection{Survivability of Attack Missile}

The survivability or Probability of Survival $P_{s}$ of the attack missile is important to be quantified to measure the effectiveness of the proposed repulsive potential function and guidance law. As introduced in [28, Ch. 1], the survivability is the complement of the killability or Probability of Kill $P_{k}$ which quantifies the probability of the aircraft being killed. Thus, the relationship can be formulated as

$$
P_{S}=1-P_{K} \text {. }
$$

From a practical point of view, the killability $P_{k}$ is a complex function, which is explained in detail in the reference [29, Ch. 6.3]. The intercept missiles typically use a fragmentation warhead, which at a particular distance to its target, detonates the explosive charge and breaks up the warhead case into smaller accelerating fragments. The killability for this fragmentation warhead is defined as a function of five parameters, i.e.: the presented target area, the vulnerable target area, the total number of fragments, spray angle of fragments, and miss distance at detonation. Recalling the reference, to achieve $P_{k}=0.9$, the number of hits on a target with a vulnerable area is minimum $10 \%$ of the presented area, which needs a minimum of 22 hits from the total fragmentation at the target.

Since all parameters in the reference are given for a rocket baseline against a drone aircraft, those assumptions can be partially adopted into this paper. The first assumption is that all the five parameters are known to achieve $P_{k}=0.9$. Secondly, the function of the killability will only depend on miss distance at detonation $d_{\mathrm{det}}$. Finally, following the typical relation trends of $P_{k}$ and $d_{\mathrm{det}}$ a simple relation is introduced as

$$
P_{K}=\exp \left(-\left(\frac{d_{\mathrm{det}}}{R_{d_{0}}}\right)^{2}\right),
$$

where $R_{d_{0}}$ is defined as the reference distance. In order to achieve the $P_{k}=0.9$ at $d_{\mathrm{det}}=6 \mathrm{~m}, R_{d_{0}}=18.5 \mathrm{~m}$ is chosen to fit the curve.

\section{Numerical Analysis}

\subsection{Performance of Proposed Guidance Law}

Let us consider a typical Suppression of Enemy Air Defenses (SEAD) mission of our attack missile with constant velocity. The attack missile is in its terminal phase maintaining the constant velocity and terminal impact angle towards the stationary target. The defending enemy's intercept missile is planted in front of the target facing the attack missile. The intercept missile will be launched at a range where the attack missile can be intercepted and neutralized in constant and $10 \%$ faster velocity than the attack missile. The intercept missile has a designated time of flight due to propellant burn time limitation and, if the time exceeds, it is considered that the intercept missile fails to neutralize the attack missile. To implement the proposed guidance law, the position of intercept missile and target are assumed to be clearly detected by the attack missile as a result of active radar or passive seeker detection. A parameter study is carried out to elaborate the performance of evasive maneuver due to design parameters gains $[\varepsilon, \zeta, \mu]$. The quantities of each parameter for the SEAD engagement scenario are listed in Table 1. 
As seen in Fig. 4, the performance of evasive maneuver is highly sensitive to the gains $[\varepsilon, \zeta, \mu]$. By defining LBF gain $\mu=0$, the performances of APF gains $[\varepsilon, \zeta]$ are evaluated on the left and center of the Fig. 4. Recalling the properties of the repulsive potential field, the steepness of the potential is verified by an early evasive maneuver when the attack missile enters the distance of influence radius $d_{0}$. With respect to the non-evasive maneuver, the evasive deviation width is determined by the value of the APF gains $[\varepsilon, \zeta]$. Starting from $\varepsilon \geq 70$ with constant $\zeta$, the attack missile is able to escape the interception and gives wider evasive trajectories for higher values. At a constant $\varepsilon=90$, wider evasive trajectories are also

Table 1. Parameters of SEAD engagement scenario

\begin{tabular}{lcl}
\hline Parameter & Symbol & Value \\
\hline Target, stationary position & $\left(x_{T}, y_{T}\right)$ & $(4,0) \mathrm{km}$ \\
Attack missile, initial position & $\left(x_{A_{0}}, y_{A_{0}}\right)$ & $(-5,6) \mathrm{km}$ \\
Intercept missile, initial position & $\left(x_{I_{0}}, y_{I_{0}}\right)$ & $(0,0) \mathrm{km}$ \\
Attack missile, velocity & $V_{A}$ & $200 \mathrm{~m} / \mathrm{s}$ \\
Intercept missile, velocity & $V_{I}$ & $220 \mathrm{~m} / \mathrm{s}$ \\
Attack missile, initial flight path angle & $\gamma_{0}$ & $-30 \mathrm{deg}$ \\
Attack missile, terminal impact angle & $\gamma_{f}$ & $-30 \mathrm{deg}$ \\
Intercept missile, time of flight & - & $20 \mathrm{~s}$ \\
Gain set of TPG & $(m, n)$ & $(2,3)$ \\
Distance of Influence of Obstacle & $d_{0}$ & $6 \mathrm{~km}$ \\
\hline
\end{tabular}

demonstrated for higher values of $\zeta$. Wide evasive trajectory maximizes the escape of the attack missile, but intuitively generates a huge flight path angle and exceeding FOV. For the values of $\zeta \geq 3 e^{-4}$, the guidance law needs a compensation command to suppress the flight path angle to ensure its seeker look angle inside FOV limit. Right graph of Fig. 4 illustrates the effect for different values of LBF gain $\mu$ which higher value of $\mu$ suppresses the guidance of attack missile for narrower path trajectory. Regarding this parameter study, gain combinations of $\varepsilon=[90,100], \zeta=3 e^{-4}, \mu=[5,10]$ are picked for further analysis since it complies with the initial requirement to achieve zero miss distance and escaping an interception.

Elaboration of look angle $\lambda$ suppression, flight path angle $\gamma$, and acceleration command $a_{S Y N}$ constraints are time series plotted in Fig. 5. Once the look angle approaching the barrier value $\lambda_{\lim }= \pm 40^{\circ}$, the LBF gains $\mu=[5,10]$ generate a suppression command to keep the look angle inside the FOV. The higher value of gain, the more suppression applied to the look angle. However, gain values $\mu \leq 2$ in this scenario are not adequate to maintain the look angle from violating the limit. From the parametric study, the value $\mu=10$ is a rational choice to satisfy both constraints and will be used for further examination. Depressed look angle when approaching FOV limit affects the flight path angle correspondingly. By implementing the IACG of TPG, the impact angle constraint is satisfied even though its basic guidance law is combined with other components by guidance synthesis. All impact angle histories are relaxed into impact angle constraint $\gamma_{0}=-$
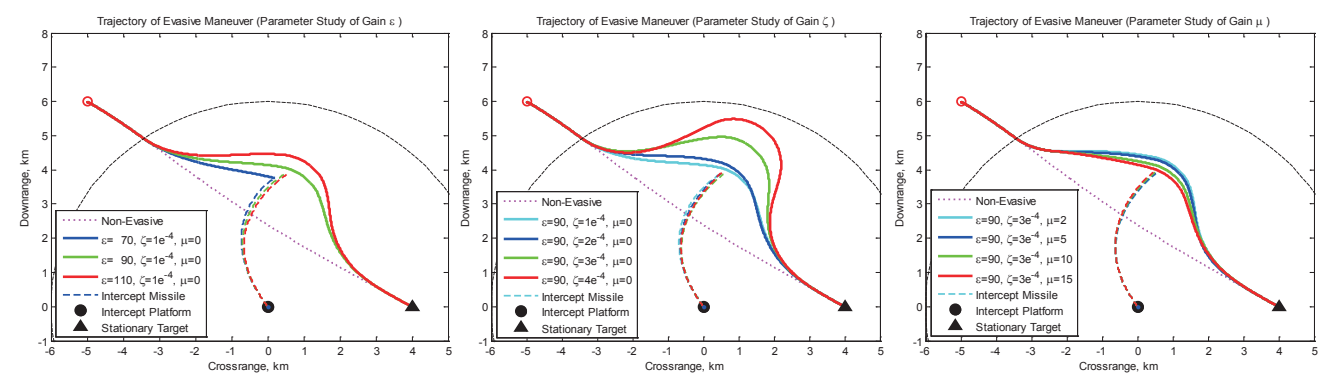

Fig. 4. Parameter study of APF gain $\varepsilon$ (left), APF gain $\zeta$ (center), and LBF gain $\mu$ (right)
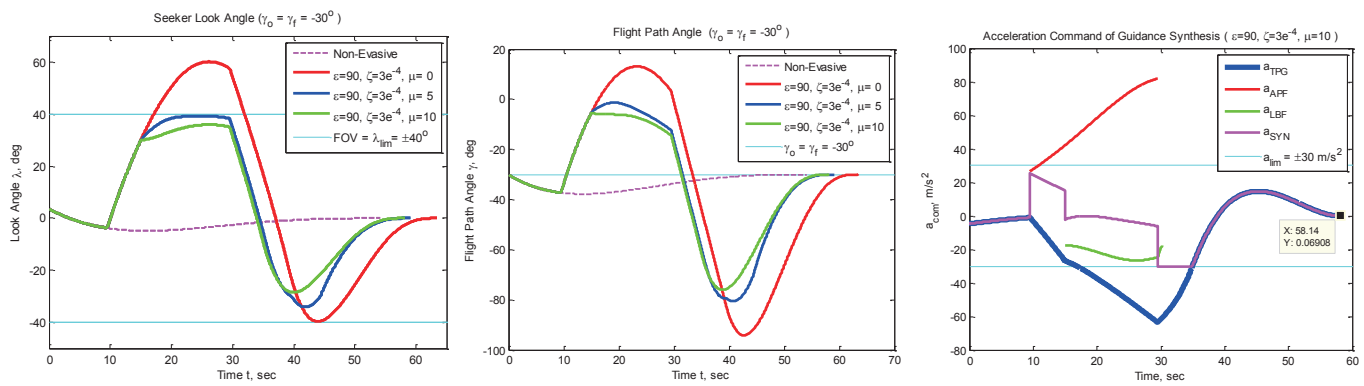

Fig. 5. Seeker look angle (left), flight path angle (center), and acceleration command (right) 

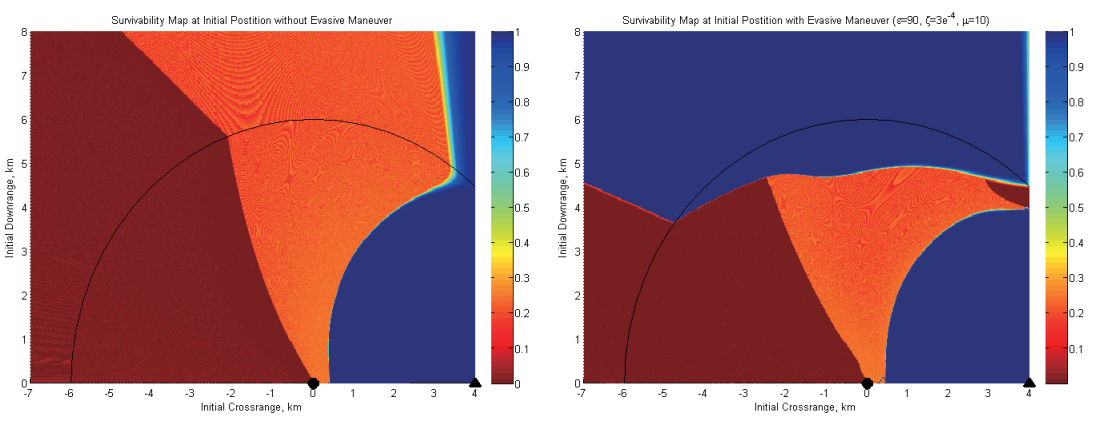

Fig. 6. Survivability map as a function of initial position without evasive maneuver (left) and with evasive maneuver (right)

$30^{\circ}$, and the look angle converges to zero. Recalling Fig. 5, components of acceleration command express the synthesis of the new guidance laws for the gain setting $\varepsilon=90, \zeta=3 e^{-4}$, $\mu=10$. The synthesized total attack command $a_{S Y N}$ effectively satisfies the terminal zero acceleration of TPG and keeps inside actuator's acceleration limit $a_{\lim }= \pm 30 m s^{2}$.

\subsection{Interception Survivability}

Applying the same engagement scenario as studied above, the attack missile is simulated at its terminal phase maintaining the prescribed impact angle, constant velocity, and launched at all possible region above target. The initial flight path angle of attack missile is determined as the initial LOS angle to the target and defined as the terminal impact angle to be achieved $\left(\gamma_{f}=\gamma_{0}=\sigma_{0}\right)$. Fig. 6 compares the survivability map of the attack missile with evasive maneuver regarding the survivability map without evasive maneuver.

The higher survivability, towards $P_{s}=1$, represents that the attack missile has more probability to survive from interception. In contrast, the lower survivability towards $P_{s}=0$ represents the attack missile has more probability to be intercepted and neutralized before hitting the target. By only implementing a single set of gain $\varepsilon=90, \zeta=3 e^{-4}, \mu=10$, the guidance law demonstrates its effectiveness to increase the survivability of the attack missile on the SEAD mission scenario. The proposed guidance law extends the area of initial position for the attack missile, which has the high survivability to accomplish the mission.

\subsection{Implementation in Operational Scenario}

Based on the parametric study and considering the survivability map, the trajectory plot in Fig. 7 elaborates three operational scenarios of broader initial position inside the initial distance of influence. Those scenarios represent a practical implementation of the guidance law based on a single gain set $\varepsilon=90, \zeta=3 e^{-4}, \mu=10$. Different initial positions, i.e.: $[-4.9,3.7] \mathrm{km},[-1.5,4.8] \mathrm{km}$, and $[1.5,5.2] \mathrm{km}$ are defined as Scenario 1, 2, and 3 respectively to represent a general engagement of SEAD scenario. In each scenario, three trajectories of simulation result are displayed, i.e.: the trajectory of attack missile with and without evasive maneuvers, and the trajectory of the intercept missile.

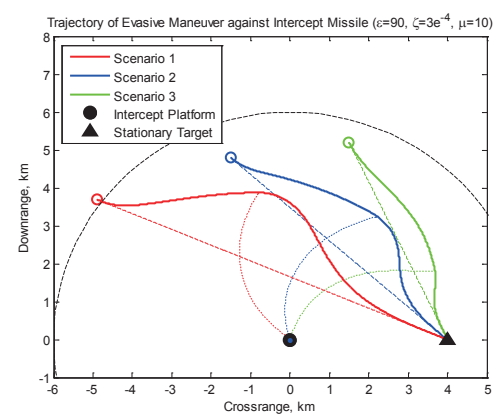

Fig. 7. Trajectory of operational scenario
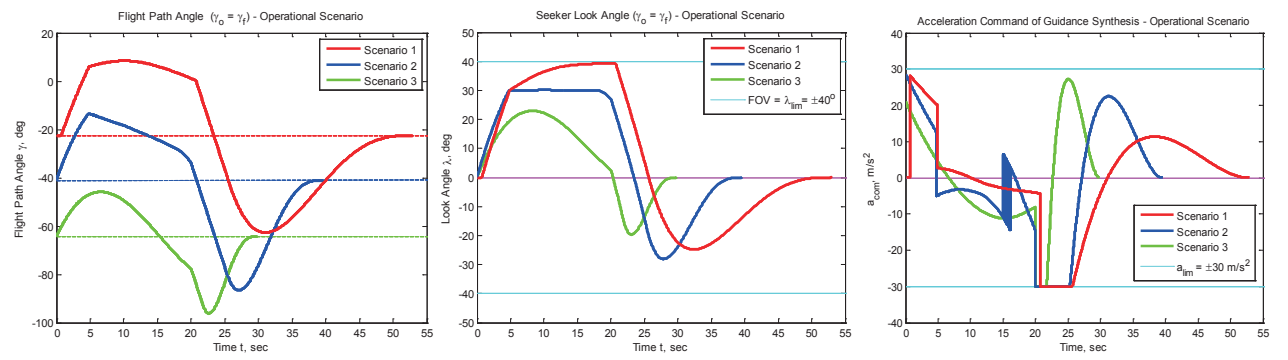

Fig. 8. Flight path angle (left), look angle (center), and acceleration command (right) of operational scenarios 
Since the engagement scenario implementing the proposed guidance law, the trajectories clearly validate the effectiveness of the guidance law to escape from interception by generating an evasive maneuver for different and wider range of initial position.

Furthermore, as seen in Fig. 8, the constraints of terminal impact angle, look angle limitation, and zero terminal acceleration are all satisfied in the three operational scenarios. Since the impact angles are defined as the initial flight path $\left(\gamma_{0}=\gamma_{f}\right)$ respectively, the terminal impact angles are all smoothly achieved in each scenario. The look angles are also suppressed to keep inside the limitation of FOV and converge to zero look angle. Finally, in addition to limit the acceleration command, the terminal accelerations are also converged to zero. All results verify that the synthesis of new guidance law based on the new proposed repulsive potential function is effective and reliable to be implemented as a new solution for evasive maneuver against intercept missiles.

\section{Conclusion}

This paper proposes a synthesis of new guidance law to generate an evasive maneuver against enemy's missile interception while considering its impact angle, acceleration, and FOV constraints. The guidance law introduces a simple approach but an effective result for a real-time avoidance against dynamic obstacles. The new guidance law synthesizes three components, starting with the new repulsive potential function of APF to generate the evasive maneuver. The zero terminal miss distance is satisfied by TPG as well as to satisfy the impact angle and zero terminal acceleration. The guidance law is finally synthesized with the LBF to guarantee the look angle inside the FOV. The parametric study on the gains of $[\varepsilon, \zeta, \mu]$ are carried to generate a reliable gain set.

SEAD engagement scenarios of attack missile headed for a stationary target that is defended by an intercept missile are performed on numerical simulation. The gain set $\varepsilon=90$, $\zeta=3 e^{-4}, \mu=10$ demonstrates the expected performance of guidance law. The commanded acceleration is proven to generate the evasive maneuver of attack missile avoiding the enemy's missile interception. The avoidance trajectory of attack missile is adequate to escape the interception while managing the constraints of impact angle, zero impact acceleration, and FOV limit. Without the evasive maneuver, the attack missile cannot survive the interception. However, the survivability of the attack missiles is enhanced when the guidance law is applied. The enhancement of survivability is clearly compared in survivability maps for the scenarios with and without evasive maneuver. Briefly, the proposed guidance law and new repulsive potential function perform a simple, reliable and effective approach to generate evasive maneuver against missile intercept while satisfying the constraints.

Study on the optimization of gain values can be carried out as further works. Optimized scaling gains will improve the guidance performance and also enhance the survivability in a broader area of initial position. An expansion of probability map using optimized gains will ensure the success of engagement mission over a high threat environment. Implementation of the proposed guidance law for multiple missiles and another engagement geometry such as air-to-air or surface-to-air scenarios are also considered for future works.

\section{Acknowledgement}

Y. H. Yogaswara expresses his appreciation to Indonesia Endowment Fund for Education (LPDP RI), for providing scholarship on a doctoral program at the Korea Advanced Institute of Science and Technology, Republic of Korea.

\section{References}

[1] Joint Chiefs of Staff., Joint Publication 3-01: Countering Air and Missile Threats, US Department of Defense, Washington DC, 2012.

[2] Zarchan, P., Tactical and Strategic Missile Guidance, 6th ed., American Institute of Aeronautics and Astronautics, Inc., Reston, Virginia, 2012.

[3] Ryoo, C. K., Whang, I. H. and Tahk, M. J., “3-D Evasive Maneuver Policy for Anti-Ship Missiles Against Close-In Weapon Systems", AIAA Guidance, Navigation, and Control Conference and Exhibit, Austin, Texas, 2003, pp. 1-7. DOI: 10.2514/6.2003-5653

[4] Kim, Y. H., Ryoo, C. K. and Tahk, M. J., "Guidance Synthesis for Evasive Maneuver of Anti-Ship Missiles Against Close-in Weapon Systems", IEEE Transactions on Aerospace and Electronic Systems, Vol. 46, No. 3, Jul. 2010, pp. 13761388. DOI: 10.1109/TAES.2010.5545195

[5] Washsberger, C., Lucas, M. and Krstic, A., Limitations of Guns as a Defence against Manoeuvring Air Weapons, DSTO Systems Sciences Laboratory, Edinburgh, South Australia, 2004.

[6] Jeon, I. S., Lee, J. I. and Tahk, M. J., "Impact-TimeControl Guidance Law for Anti-Ship Missiles", IEEE Transactions on Control Systems Technology, Vol. 14, No. 2, Mar. 2006, pp. 260-266. DOI: 10.1109/TCST.2005.863655

[7] Shiyu, Z., Rui, Z., Chen, W. and Quanxin, D., "Design 
of Time-Constrained Guidance Laws via Virtual Leader Approach", Chinese Journal of Aeronautics, Vol. 23, No. 1, 2010, pp. 103-108. DOI: 10.1016/S1000-9361(09)60193-X

[8] Cho, D., Kim, H. J. and Tahk, M. J., "Nonsingular Sliding Mode Guidance for Impact Time Control", Journal of Guidance, Control, and Dynamics, Vol. 39, No. 1, 2015, pp. 61-68. DOI: 10.2514/1.G001167

[9] Saleem, A. and Ratnoo, A., "Lyapunov-Based Guidance Law for Impact Time Control and Simultaneous Arrival Abdul", Journal of Guidance, Control, and Dynamics, Vol. 39, No. 1, 2016, pp. 164-172. DOI: 10.2514/1.G001349

[10] Krogh, B. H., "A Generalized Potential Field Approach to Obstacle Avoidance Control", Robotics Research: The Next Five Years and Beyond, Bethlehem, Pennsylvania, 1984.

[11] Khatib, O., "Real-Time Obstacle Avoidance for Manipulators and Mobile Robots", The International Journal of Robotics Research, Vol. 5, No. 1, Mar. 1986, pp. 90-98. DOI: 10.1177/027836498600500106

[12] Koren, Y. and Borenstein, J., "Potential Field Methods and Their Inherent Limitations for Mobile Robot Navigation", Proceedings of 1991 IEEE International Conference on Robotics and Automation, 1991, pp. 1398-1404. DOI: 10.1109/ ROBOT.1991.131810

[13] Ge, S. S. and Cui, Y. J., "New Potential Functions for Mobile Robot Path Planning", IEEE Transactions on Robotics and Automation, Vol. 16, No. 5, 2000, pp. 615-620. DOI: $10.1109 / 70.880813$

[14] Ge, S. and Cui, Y., “Dynamic Motion Planning for Mobile Robots Using Potential Field Method", Autonomous Robots, Vol. 13, No. 3, 2002, pp. 207-222. DOI: 10.1023/A:1020564024509

[15] Park, D. H., Hoffmann, H., Pastor, P. and Schaal, S., "Movement Reproduction and Obstacle Avoidance with Dynamic Movement Primitives and Potential Fields", Humanoids 2008 - 8th IEEE-RAS International Conference on Humanoid Robots, 2008, No. 1, pp. 91-98. DOI: 10.1109/ ICHR.2008.4755937

[16] Qixin, C., Yanwen, H. and Jingliang, Z., "An Evolutionary Artificial Potential Field Algorithm for Dynamic Path Planning of Mobile Robot", 2006 IEEE/RSJ International Conference on Intelligent Robots and Systems, Vol. 71, 2006, pp. 3331-3336. DOI: 10.1109/IROS.2006.282508

[17] Chen, Y. B., Luo, G. C., Mei, Y. S., Yu, J. Q. and Su, X. L., "UAV Path Planning Using Artificial Potential Field Method Updated by Optimal Control Theory", International Journal of Systems Science, Vol. 47, No. 6, Apr. 2016, pp. 1407-1420. DOI: 10.1080/00207721.2014.929191

[18] Ryoo, C. K., Cho, H. and Tahk, M. J., "Optimal Guidance Laws with Terminal Impact Angle Constraint", Journal of Guidance Control and Dynamics, Vol. 28, No. 4,
2005, pp. 724-732. DOI: 10.2514/1.8392

[19] Ratnoo, A. and Ghose, D., "Impact Angle Constrained Interception of Stationary Targets", Journal of Guidance Control and Dynamics, Vol. 31, No. 6, 2008, pp. 1816-1821. DOI: $10.2514 / 1.37864$

[20] Kim, M. and Kim, Y., "Lyapunov-Based Pursuit Guidance Law with Impact Angle Constraint", 19th World Congress The International Federation of Automatic Control, Cape Town, South Africa, 2014, pp. 2509-2514. DOI: 10.3182/20140824-6-ZA-1003.00233

[21] Kim, H. and Kim, H. J., "Missile Guidance Law Considering Constraints on Impact Angle and Terminal Angle of Attack", AIAA Guidance, Navigation, and Control Conference, Kissimmee, Florida, 2015, pp. 1-16. DOI: 10.2514/6.2015-0861

[22] Lee, C. H., Kim, T. H., Tahk, M. J. and Whang, I. H., "Polynomial Guidance Laws Considering Terminal Impact Angle and Acceleration Constraints", IEEE Transactions on Aerospace and Electronic Systems, Vol. 49, No. 1, 2013, pp. 7492. DOI: 10.1109/TAES.2013.6404092

[23] Sang, D. K. and Tahk, M. J., "Guidance Law Switching Logic Considering the Seeker's Field-of-View Limits", Proceedings of the Institution of Mechanical Engineers, Part G: Journal of Aerospace Engineering, Vol. 223, No. 8, 2009, pp. 1049-1058. DOI: 10.1243/09544100JAERO614

[24] Zhang, Y., Wang, X. and Wu, H., "Impact Time Control Guidance Law with Field of View Constraint", Aerospace Science and Technology, Vol. 39, 2014, pp. 361-369. DOI: 10.1016/j.cja.2013.04.037

[25] Park, B. G., Kim, T. H. and Tahk, M. J., “Optimal Impact Angle Control Guidance Law Considering the Seeker's Fieldof-View Limits", Proceedings of the Institution of Mechanical Engineers, Part G: Journal of Aerospace Engineering, Vol. 227 , No. 8, 2012, pp. 1347-1364. DOI: 10.1177/0954410012452367

[26] Wang, X., Zhang, Y. and Wu, H., "Sliding Mode Control Based Impact Angle Control Guidance Considering the Seeker's Field-of-View Constraint", ISA Transactions, Vol 61, 2015, pp. 49-59. DOI: 10.1016/j.isatra.2015.12.018

[27] Shukla, U. S. and Mahapatra, P. R., "The Proportional Navigation Dilemma-Pure or True?", IEEE Transactions on Aerospace and Electronic Systems, Vol. 26, No. 2, Mar. 1990, pp. 382-392. DOI: 10.1109/7.53445

[28] Ball, R. E., The Fundamentals of Aircraft Combat Survivability: Analysis and Design, 2nd ed., American Institute of Aeronautics and Astronautics, Inc., Reston, Virginia, 2003.

[29] Fleeman, E. L., Missile Design and System Engineering, 1st ed., American Institute of Aeronautics and Astronautics, Inc., Reston, Virginia, 2012. 
2017-09-11

\section{Impact angle control guidance synthesis for evasive maneuver against intercept missile}

Yogaswara, Y. H.

Korean Society for Aeronautical and Space Sciences

Yogaswara YH, Hong S-M, Tahk M-J, Shin H-S, Impact angle control guidance synthesis for evasive maneuver against intercept missile, International Journal of Aeronautical and Space Sciences, Vol. 18, Issue 4, 2017, pp. 719-728

http://dx.doi.org/10.5139/IJASS.2017.18.4.719

Downloaded from Cranfield Library Services E-Repository 